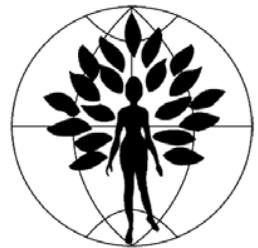

\title{
Sexual function and sexual health: New approaches to an old concern
}

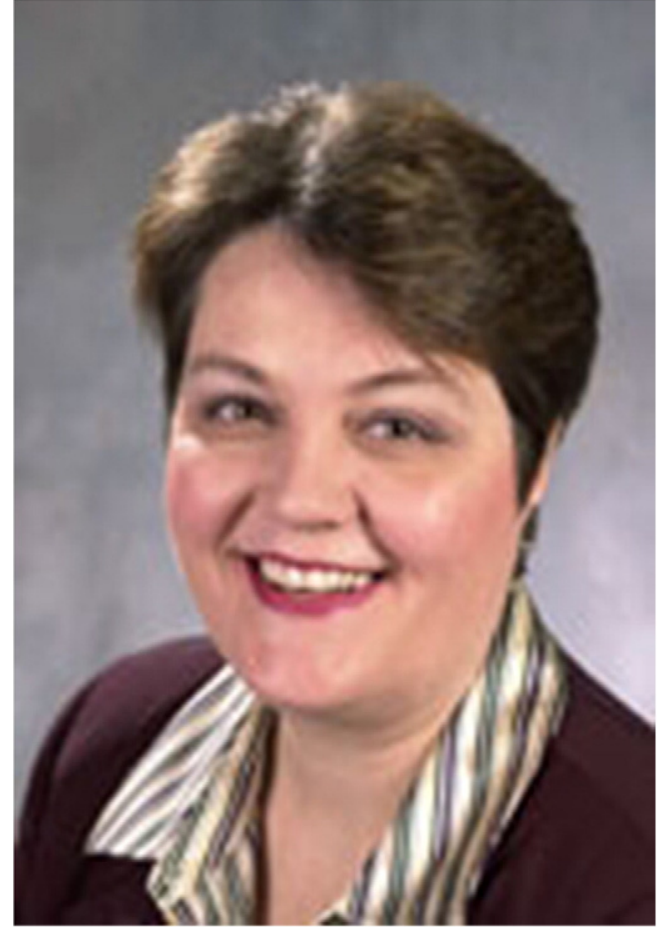

Dee Fenner, M.D.

University of Michigan, Ann Arbor, USA

Dr Dee Fenner is the Furlong Professor of Women's Health, the Director of Gynecology, and the Associate Chair for Surgical Services in the Department of Obstetrics and Gynecology at the University of Michigan, Ann Arbor, USA. Dr Fenner attended undergraduate and medical school at the University of Missouri, Columbia, and completed a residency in Obstetrics and Gynecology at the University of Michigan followed by a fellowship in Pelvic Reconstructive Surgery and Urogynecology at the Mayo Clinic, Scottsdale. Dr Fenner served as the Director of Medical Student Education and as the Residency Director at Rush Medical College in Chicago for several years and as the Vice-Chair for Education in the Department of Obstetrics and Gynecology at the University of Washington. In 2001, Dr Fenner returned to the University of Michigan where she continues her work in female pelvic floor dysfunction and medical education. She is nationally recognized as an expert in defecation disorders, surgical education, and reconstructive surgery, and has published more than 50 articles and chapters. Dr Fenner co-edits the series "Surgery and Technology" in the International Journal of Gynecology and Obstetrics.

Obstetricians and gynecologists, urogynecologists, and other health care providers caring for women with pelvic floor dysfunction have classically focused on bladder and bowel control problems and pelvic organ prolapse. The evaluation, treatment, and research aimed at understanding the underlying pathophysiology of these conditions have centered on these 3 domains of pelvic floor function. However, in the last 5 years, we have seen a fourth domain added, that of sexual function [1].

Sexual function and female sexual dysfunction (FSD), as related to other pelvic floor conditions, are of vital importance for two major reasons. First, sexual function can be greatly impacted by other pelvic conditions, such as urinary incontinence or pelvic organ prolapse. Pelvic floor disorders may lead to FSD, the disturbance in sexual desire and psychophysiological changes that characterize the sexual response and cause marked distress and personal difficulty. The key to determining FSD is measuring the distress and personal difficulty to sexual function. Lack of sexual activity or desire may not cause distress for all women. Yet fear of urinary incontinence with intercourse, loss of self-esteem secondary to urine or bowel leakage, and pain or discomfort secondary to vaginal prolapse can greatly impact a woman's desire for sexual activity and comfort during intercourse, and can lead to FSD [2]. Second, treatments, including pharmacotherapy, pessaries, and surgeries can impact sexual function and FSD in a positive or negative way [3]. Clinicians caring for women with pelvic floor dysfunction need to be aware of these interactions when forming treatment plans and should discuss the possible benefits and risks of different therapies on sexual function.

Sexuality, sexual response, and sexual function are a result of complex psychological, social, neurological, and 
anatomical interactions that are difficult to study. Isolating specific links to other disease processes, such as urinary incontinence, are very challenging. The reported prevalence of FSD in female populations worldwide ranges from $20 \%$ to $50 \%$ and in women with urinary incontinence the prevalence of FSD is as high as $47 \%$.

The two papers in this issue of the IJGO-one from France by Berthier et al. [4] on "Sexual function in women following the transvaginal tension-free tape procedure for incontinence," and one from the United Kingdom by Thaker et al. [5] on "Sexual function following pelvic floor surgery"-focus our attention on the impact surgical treatments may have on sexual function. The articles are similar in that they aim to detect change in sexual function following surgical interventions and, as is common in this field of study, they differ in the methods and questionnaires used to assess sexual function $[6,7]$.

Berthier et al. [4] performed a retrospective chart review to identify women who underwent a tension-free vaginal tape (TVT) procedure for stress urinary incontinence. A nonvalidated but often used questionnaire was sent via postal service to 135 women. Sixty-six questionnaires were reported on for an analyzable response rate of $50 \%$. Mean follow-up was $38 \pm 15.5$ months. Overall, $70 \%$ of the women were not sexually active before and after surgery because of lack of a partner. One woman who was not sexually active before surgery because of urinary incontinence with intercourse became sexually active after the procedure. Half of the women reported no change in intercourse following the surgery, 25\% reported that intercourse was worse, and $25 \%$ reported that intercourse was better. This study is consistent with the literature, nicely reviewed in the paper, which is equally split on the improvement or decline in sexual function following TVT. For some women, decrease in the fear of incontinence and rise is self-esteem and quality of life with resolution of incontinence may result in an improvement in sexual function. For other women, possibly due to pain, proximity of the tape to clitoral branches of the pudendal nerve, or erosion of the mesh tape, coital sexual pleasure could decline. My take home message from this paper, and in reviewing the other literature, is that we must council women that a TVT or other midurethral sling may positively or negatively impact sexual function. However, the majority of women will see no impact. Talking with the patient about her sexual activity, fears, orgasmic function, and how she relates her incontinence to sexual function before surgery is imperative.

Thacker et al. [5] performed a prospective study of 35 women undergoing prolapse and urinary incontinence surgeries who were queried before and 4 months after surgery. The study used the validated short-form Pelvic Organ Prolapse/Urinary Incontinence Sexual Questionnaire (PISQ) and found an overall improvement in sexual function with statistical increases in the overall score and the physical- and partner-related domains. In addition, the study evaluated the reliability and performance of this questionnaire, developed in the United States, in a different culture and population. The authors point out the importance of cultural norms and language when discussing sexual practices. In their population the question addressing masturbation was not completed by a majority of women. In addition, there was poor reliability found for the partner-related section. In summary, while the women showed improvement in sexual function as demonstrated by the change in preoperative and postoperative scores, the degree of distress related to sexual dysfunction and the factors that determined improvement after surgery could not be determined.

These articles are excellent examples of the new and vital work being done to evaluate the domain of sexual function and the pelvic floor. It is hoped that this expanding body of knowledge, using culturally specific validated questionnaires, will answer many questions concerning relationships between anatomy and function of the pelvic floor and sexual function. In addition, an increasing concern is the rise in permanent mesh materials being used for surgical management of urinary incontinence and pelvic organ prolapse, and the impact the mesh will have on sexual function must be explored [8]. Clinicians, surgeons, product manufacturers, and patients must be diligent and thoughtful in evaluating and reporting the benefits and risks of new and old treatments for pelvic floor disorders on sexual function and sexual health.

\section{References}

[1] Dalpiaz O, Kerschbaumer A, Mitterberger M, Pinggera GM, Colleselli D, Bartsch G, et al. Female sexual dysfunction: a new urogynaecological research field. BJU Int 2008;101(6):717-21.

[2] Shaw C. A systematic review of the literature on the prevalence of sexual impairment in women with urinary incontienence and the prevalence of urinary leakage during sexual activity. Eur Urol 2002;42(5):432-40.

[3] Pauls RN, Segal JL, Silva WA, Kleeman SD, Karram MM. Sexual function in patients presenting to a urogynecology practice. Int Urogynecol J Pelvic Floor Dysfunct 2006;17(6):576-80.

[4] Berthier A, Sentilhes L, Taibi S, Loisel C, Grise P, Marpeau L. Sexual function in women following the transvaginal tensionfree tape procedure for incontinence. Int J Gynecol Obstet 2008;102(2) [complete at issue compile].

[5] Thakar R, Chawla S, Scheer I, Barrett G, Sultan AH. Sexual function following pelvic floor surgery. Int J Gynecol Obstet 2008;102(2) [complete at issue compile].

[6] Rogers RG, Coates KW, Kammerer-Doak D, Khalsa S, Qualls C. A short form of the Pelvic Organ Prolapse/Urinary Incontinence Sexual Questionnaire (PISQ-12). Int Urogynecol J Pelvic Floor Dysfunct 2003;14(3):164-8.

[7] Laumann EO, Nicolosi A, Glasser DB, Paik A, Gingell C, Moreira E, et al. Sexual problems in women and men $40-80 \mathrm{y}$ : prevalence and correlates identified in the Global Study of Sexual Attitudes and Behaviors. Int J Impot Res 2005;17(1):39-57.

[8] Huebner M, Hsu Y, Fenner DE. The use of graft materials in vaginal pelvic floor surgery. Int J Gynecol Obstet 2006;92(3):279-88.

Dee Fenner

University of Michigan, Ann Arbor, USA E-mail address: deef@med.umich.edu. 